\title{
A Method for Image Mosaic of Textile Printing Fabric Based on SIFT Feature Matching
}

\author{
Suyi Liu
}

College of Mechanical Engineering and automation Wuhan Textile University Wuhan, 430073, China

liusuyi@sina.com

Keywords: SIFT; Image mosaic; Feature matching; Textile printing fabric

\begin{abstract}
Image mosaic is the technique to stitch a series of overlapped pictures into a bigger one. The quality of image mosaic mainly depends on the precision of image registration. In the process of image mosaic the features should be acquired and matched accurately. Recently, SIFT (Scale Invariant Feature Transform) feature matching algorithm is becoming the focus of the image mosaic. In this paper focuses on the point matching of the textile fabric by SIFT. As is known to all, in the detection of textile fabrics, the image acquisition is the key, Based on this, this article through the SIFT algorithm for textile printing image stitching, get a picture of a wide range of complete image.
\end{abstract}

\section{Introduction}

SIFT[1] (scale invariant feature transform) is a method proposed by Lowe in 2004 based on the scale space, it is an image local feature description operator can keep invariance when zoom, rotation and affine transformation of the image, it can be used to extract the scale invariant feature from different perspectives images for image matching and object recognition. Therefore, the algorithm that based on SIFT characteristics has been widely used in many areas such as image mosaic[2], image registration and so on nowadays. The work of this paper is to apply the SIFT algorithm to the textile printing cloth image mosaic.

\section{SIFT Algorithm}

The SIFT algorithm mainly has the following steps:

Step1: Construct Scale Space. SIFT algorithm is based on the theory of image scale space, image scale space function is $L(x, y, \sigma)$, the input image is $\boldsymbol{I}(\boldsymbol{x}, \boldsymbol{y})$, the image $I(x, y)$ and a scale variable Gauss function $G(x, y, \sigma)$, through the convolution operation to get the scale space[3].

$$
\begin{aligned}
& L(x, y, \sigma)=G(x, y, \sigma) * I(x, y) \\
& G(x, y, \sigma)=\frac{1}{2 \pi \sigma^{2}} e^{-\left(x^{2}+y^{2}\right)} / 2 \sigma^{2}
\end{aligned}
$$

$\sigma$ is the scale parameter, corresponding to the variance of Gauss kernel function, the smaller the value, the smaller the image is smoothed and the corresponding scale is smaller.

Relationship between the scale and the image is large scale corresponding to the general features of the image, small scale corresponding to image details.

Step2: Constructing the Gauss Differential Scale Space. In order to detect stable feature points in scale space, when detecting feature points, SIFT algorithm make the adjacent two scale images to subtract each other, and get the DOG (Difference of Gauss) image, i.e. 


$$
\begin{aligned}
& D O G=G_{\sigma 1}-G_{\sigma 1} \\
& =\frac{1}{\sqrt{2 \pi}}\left(\frac{1}{\sigma_{1}} e^{-\left(x^{2}+y^{2}\right) / 2 \sigma_{1}^{2}}-\frac{1}{\sigma_{2}} e^{-\left(x^{2}+y^{2}\right) / 2 \sigma_{2}^{2}}\right)
\end{aligned}
$$

DoG scale space[4]is shown in Fig. 1

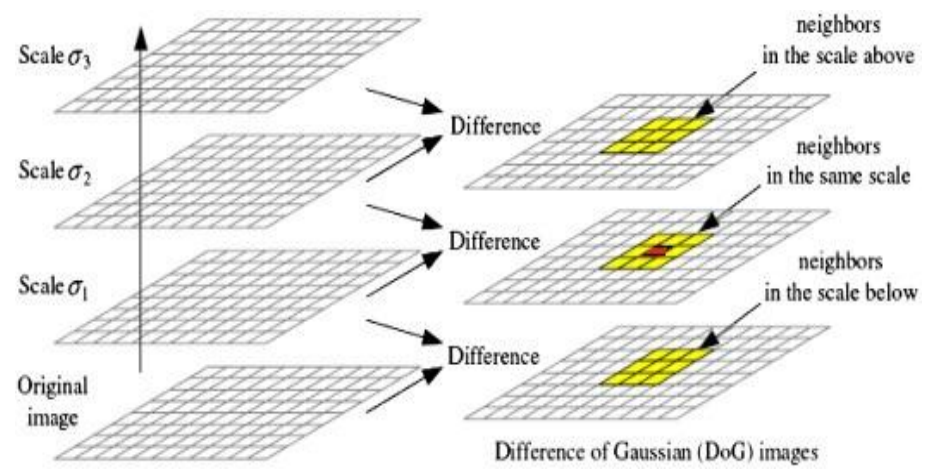

Figure 1. Gauss differential scale space

Step3: Extract Feature Points. In the adjacent three layer image of the DOG image space, that is, in a $3 \times 3 \times 3$ cube $(1+8+9+9=27$ pixels $)$ to find the maximum or minimum values, the extreme points is the feature points.

For example, in Fig. 1, the detection point (red label) is compared to 8 consecutive points with the same scale and 18 points upper and lower adjacent scales around it, deciding whether a point is extreme point, if it is, consider the detection point is a feature point. So in turn, can accomplish image feature extraction[5].

Step4: Feature Points Direction Distribution. Utilize the characteristic of the neighborhood pixel gradient direction distribution of the feature points, we can specify the direction of direction parameters for each feature point. The method is taking the feature point as the origin, using the gradient of all pixel in this area to form a direction histogram.

Determining the gradient direction of a point has the following formula:

$$
\begin{aligned}
& m(x, y)=\sqrt{\left(L(x+1, y)-\left(L(x-1, y)^{2}+L(x, y+1)-\left(L(x, y-1)^{2}\right)\right.\right.} \\
& \boldsymbol{\theta}(\boldsymbol{x}, \boldsymbol{y})=\arctan \frac{\boldsymbol{L}(\boldsymbol{x}+\mathbf{1}, \boldsymbol{y})-(\boldsymbol{L}(\boldsymbol{x}-\mathbf{1}, \boldsymbol{y})}{\boldsymbol{L}(\boldsymbol{x}, \boldsymbol{y}+\mathbf{1})-(\boldsymbol{L}(\boldsymbol{x}, \boldsymbol{y}-\mathbf{1})}
\end{aligned}
$$

The $m(x, y)$ is the gradient size at the point $(\mathrm{x}, \mathrm{y}), \theta(x, y)$ is the direction of the gradient at the point.

Sampling in the neighborhood window of the feature points as the center, and using histogram to do statistics the gradient direction of neighborhood pixels, the range of the gradient histogram is 0 360 degrees, 10 degrees each as a column, the total is 36 columns, select the direction of the largest ordinate values in the histogram as the main direction of the key point. It is shown in Fig. 2.

Step5: Generate Feature Point Descriptor. through the above steps we have found the position, scale and direction of the SIFT feature points, next we need a set of vectors to describe the key points, that is to generate feature point descriptor, the specific approach is: rotate the axis as the direction of the feature point, using the gradient amplitude and the direction of the pixel in the $16 \times 16$ window of feature point as the center, to divide the pixel in the window into 16 blocks, each block is the pixel histogram statistics of 8 direction, altogether formed 128 dimension feature vector, and normalized feature vector length. 


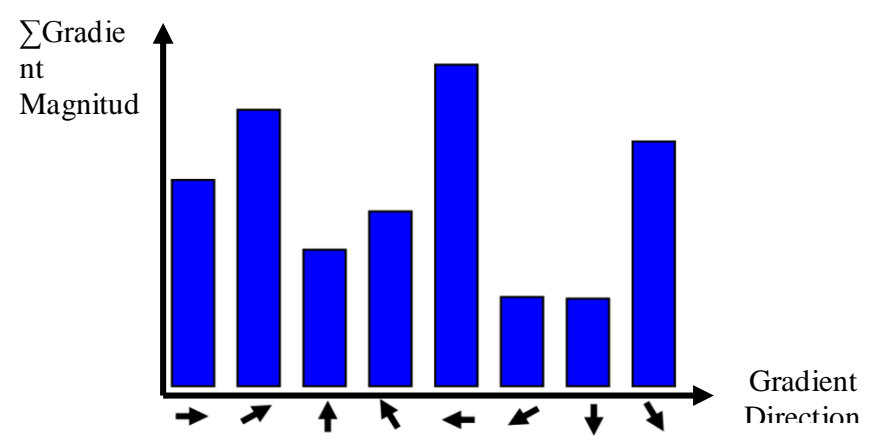

Figure 2. Histogram of feature points

\section{Image Matching Process Based on SIFT Algorithm}

The process of using SIFT algorithm to match objects image is shown in Fig. 3.

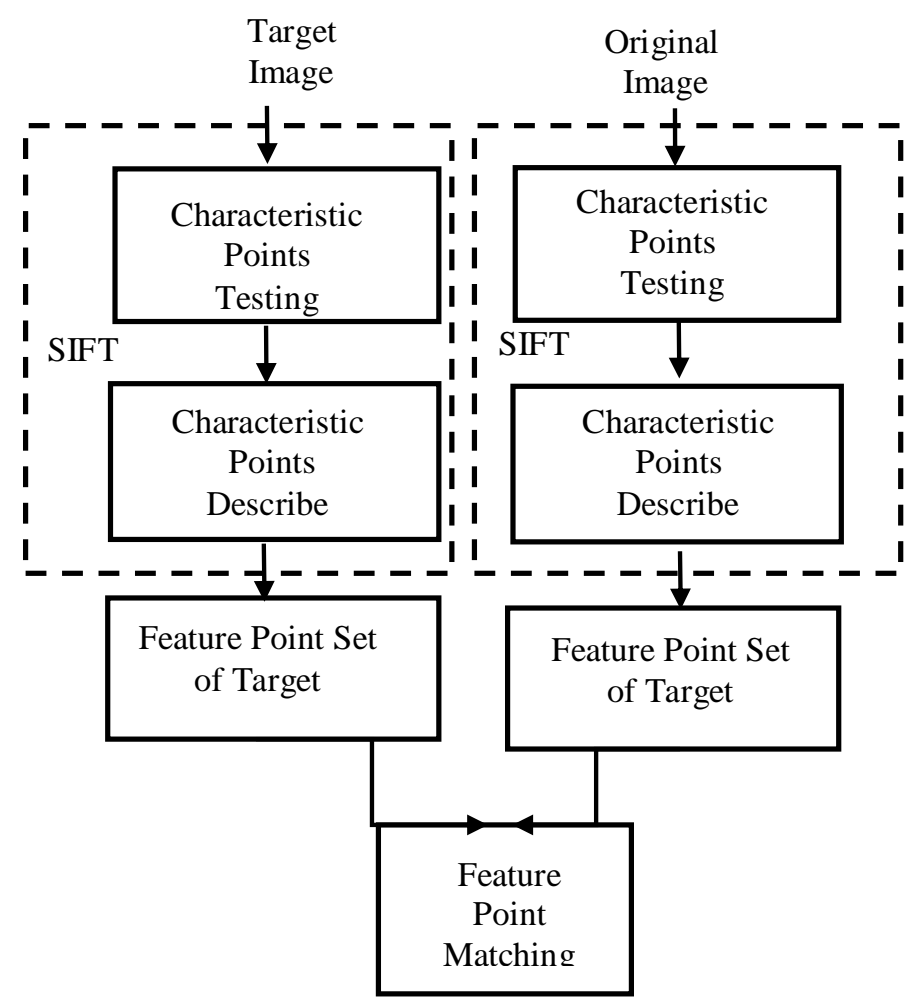

Figure 3. Image matching process based on SIFT algorithm

After generating the feature vectors of the two images, using the key points feature vectors Euclidean distance[6] as the similarity measurement of the key points of the two images. Take a key point in the image, and find out the nearest two key points in the other image, in these two key points, if the nearest distance divided by the close distance is less than a threshold value $\mathrm{T}$, then accept it as the matching point. The threshold $\mathrm{T}$ is $0.8[1]$.

To reduce this threshold, the number of matching points will be reduced, but the matching process is more stable, and on the contrary, the number of matching points will increase, but the stability will be slightly worse.

\section{Registration and Stitching the Printing Fabric Based on SIFT Algorithm}

Fabric defects are often produced in printing and dyeing, so the cloth need to be detected after printing and dyeing, if the cloth width is large, it needs image mosaic. Fig. 4 is given two pieces of cloth pattern of tomato. 

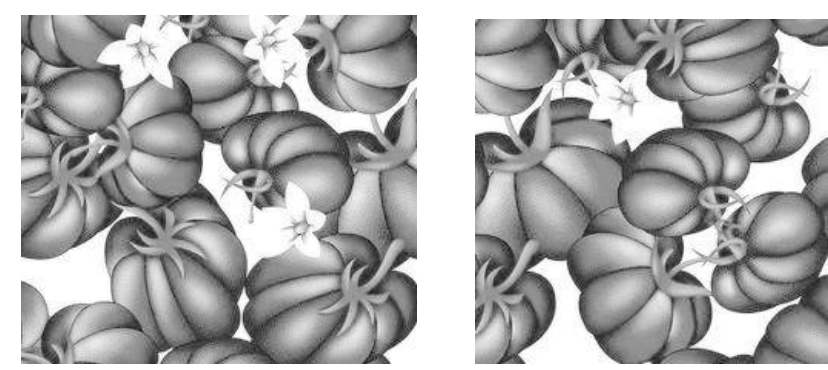

Figure 4. Two pieces of cloth pattern of Tomato

According to the above SIFT algorithm for these two images registration and stitching, shown in Fig. 5 to Fig. 7.
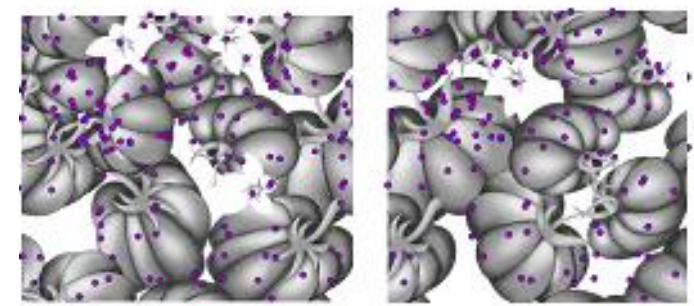

Figure 5. Feature points in detection

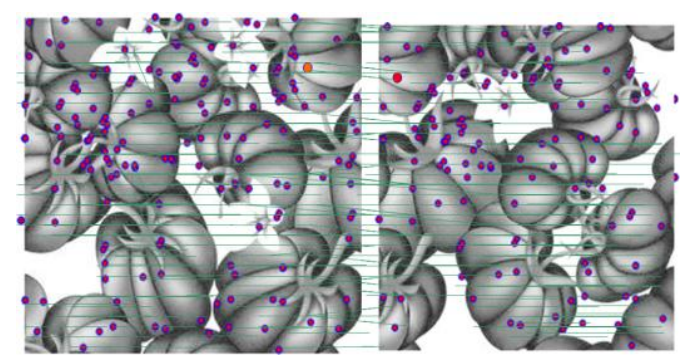

Figure 6. Feature points in image matching

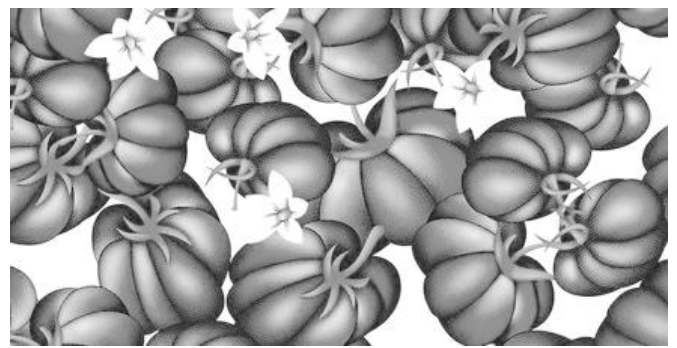

Figure 7. Image mosaic

Through the above steps it can be completed that two pieces of printing cloth image stitched perfectly, lay the foundation for the next step of the cloth detection.

\section{Conclusion}

SIFT feature matching algorithm is hot and difficult in the field of feature points matching research domestic and abroad. Its match ability is strong, and can be dealt with translation, rotation, affine transformation circumstances matching problem between the two images.

According to the SIFT feature point extraction step and precise positioning method, and applied it to the image matching of printing cloth. The experimental results show that, for textile image, using SIFT algorithm can realize image matching and stitching effectively. 


\section{References}

[1] Lowe, D. G. Distinctive image features from scale-invariant keypoints. International Journal of Computer Vision, 60(2):91-110, 2004.

[2] SUZUKI T. Vision based localization of a small UAV for generating a large mosaic images[C].IEEE SICE Annual Congerence,Taiwan,2010(10):2960-2964

[3] LINDEBERG T P. Internal report TRI TA-NA-P8808[R].Stockholm, Sweden: Royal Institute of Technology, 2000.

[4] Lindberg T. Scale-space theory: a basic tool for analyzing structures at different scales. Journal of Applied Statistics, 1994,21(2):224-270.

[5] BROWN M, LOWL, D G. Automatic panoramic image stitching using

[6] invariant features ) ].International Journal of Computer Vision,2007,4(1):59-73.

[7] GAO Feng, WEI Shao-hua, WANG Xue-tong.Image Matching Algorithm Based on SIFT Feature Point[J] The modern electronic technology,2010(18):101-103 